\title{
Neuroanatomical studies on bipolar disorder
}

\author{
BRUNO BAUMANN and BERNHARD BOGERTS
}

\begin{abstract}
Background Neuroimaging data showing structural and functional brain abnormalities in mood disorders suggest that brain alterations at the neurohistological level may underlie the macropathology seen by imaging in vivo.
\end{abstract}

Aims To summarise recent postmortem studies on affective disorders, with a focus on bipolar disorder.

\begin{abstract}
Method Literature review and discussion of results from volumetric, cyto-architectural and immunohistochemical analyses.
\end{abstract}

Results Basal ganglia are smaller in patients with depression irrespective of diagnostic polarity. In addition, higher neuron numbers have been reported in the locus caeruleus of patients with bipolar disorder compared with those with unipolar depression. Patients with bipolar as well as unipolar illness show subtle structural deficits in the dorsal raphe. Histological data are consistent with a regional reduction in the synthesis of noradrenalin and serotonin, which appears to be compensated by antidepressants.

\section{Conclusion Preliminary results suggest that, aside from functional dysregulation, subtle structural abnormalities in the brain may contribute to the pathogenesis of mood disorders.}

\section{Declaration of interest This paper}

was supported by the Deutsch Forschungsgemeinschaft (79916-I) and the Theodore and Vada Stanley Foundation.
In contrast to schizophrenia, little work has been done on the neurohistology of mood disorders, indicating that this kind of research strategy is still in its infancy, and the results obtained from these studies provide only a fragmentary pattern. Most of the studies include subjects who had committed suicide, and a retrospective diagnosis of a mood disorder was not established in all cases. Some investigations did not focus on affective disorders, but used subjects with mood disorders as a control group. Since this article emphasises regional neurohistology, biochemical studies performed on homogenate of brain tissue are not considered in the following survey.

\section{POST-MORTEM STUDIES}

\section{Studies on brain structure}

One early post-mortem study found a greater brain weight, a thicker parahippocampal cortex and smaller temporal horns in patients with affective disorder compared with those with schizophrenia (Brown et al, 1986), suggesting that temporal lobe structure differs in these disorders. Unfortunately, no control group without neuropsychiatric disorder was included in this study. Therefore, it is difficult to compare these data with magnetic resonance imaging (MRI) studies that report smaller entorhinal cortex sizes in schizophrenia, but not in bipolar disorder (Pearlson et al, 1997). It is conceivable, however, that a structural disturbance of the parahippocampal cortex is present in mood disorders, since one post-mortem study showed a reduction in size of this cortex in suicide victims compared with non-psychiatric controls (Altshuler et al, 1990). Further evidence for a disturbed morphological integrity of this region comes from cyto-architectural studies reporting that malformations in the entorhinal lamination were observed in patients with bipolar disorder or with major depression (Beckmann \& Jakob, 1991; Bernstein et al, 1998). In addition, one case report described a temporo-occipital cortical dysplasia associated with rapid-cycling bipolar disorder and learning disability (Raghavan et al, 1995). These studies suggest that both bipolar and major depressive mood disorder might be associated with circumscribed neurodevelopmental disturbances in temporal regions.

Another alteration in the temporal lobe was reported by Benes and co-workers who showed a selective reduction of nonpyramidal neurons in the CA2 region on the hippocampus of patients with bipolar disorder and schizophrenia (Benes et al, 1998). These studies confirm and extend recent MRI findings suggesting a temporal pathology not only in schizophrenia but also in bipolar disorder (Altshuler et al, 1998; Roy et al, 1998; Strakowski et al, 1999).

The prefrontal lobe is another focus of interest in mood disorders (Goodwin, 1997). Decreased cortical and laminar thickness but unchanged overall neuronal density and laminar density were demonstrated in the dorsolateral prefrontal cortex in both bipolar disorder and major depressive disorder. In contrast to patients with schizophrenia, who also showed smaller cortical thickness but an increased neuronal density in this area, patients with mood disorders appear to have unchanged neuronal densities in this part of the prefrontal cortex (Rajkowska, 1997). Also relevant to mood disorders, theoretically speaking, are the medial prefrontal cortex and the anterior cingulate cortex. Drevets and colleagues found a volume reduction and fewer glial (but no neuronal) cells in the subgenual prefrontal cortex - which is part of the anterior cingulate cortex - in familial mood disorders (Drevets et al, 1998). No differences were described between bipolar disorder and unipolar depression. In another post-mortem study a layer-specific reduction of interneurons in the anterior cingulate cortex was observed in subjects with bipolar disorder (Vincent et al, 1997). This is consistent with the finding of a deficit in a neuronal subpopulation detected by immunoreactivity for the calcium-binding protein calretinin (Diekmann et al, 1998). These results confirm and extend previous neuroimaging work revealing structural and functional abnormalities of certain parts of the prefrontal cortex in mood disorders (Goodwin, 1997).

\section{Studies on monaminergic systems}

Some neurohistological studies investigated the role of monaminergic systems in mood 
disorders. The serotonergic system was analysed in one study reporting increased binding of $5-\mathrm{HT}_{1 \mathrm{~A}}$ autoreceptors in the midbrain of subjects with major depression who had committed suicide, suggesting reduced activity of dorsal raphe neurons (Stockmeier et al, 1998). Another investigation on presynaptic markers showed unchanged serotonin transporter binding and mRNA levels in the midbrain, hippocampus or frontal cortex in cases of suicide following major depression (Little et al, 1997). Stockmeier et al (1997) found that the $5-\mathrm{HT}_{1 \mathrm{~A}}$ and the $5-\mathrm{HT}_{2 \mathrm{~A}}$ receptors were unchanged in the right frontopolar cortex. Thus some, but not all, post-mortem studies support the indolaminergic hypothesis of depression. This is in line with an imaging study indicating decreased density of serotonin transporter binding sites in the midbrain of patients with major depression (Malison et al, 1998). These data on living patients contrast with the negative postmortem findings in suicide victims with major depression (Little et al, 1997). Taken together, some post-mortem and neuroimaging studies reveal alterations in serotonergic neurons in major depression. It remains unclear whether these results, if replicable, can be generalised for bipolar disorder.

As yet, no investigation has focused on the cyto-architecture of monaminergic neuron systems, such as the raphe nuclei or the locus caeruleus, in mood disorders. An incidental note on a geriatric case of depression mentioned a considerable lack of locus caeruleus neurons (Chan-Palay \& Asan, 1989), which is in agreement with a study showing fewer neurons in this crucial noradrenergic nucleus in suicide victims (Arango et al, 1996). This needs further confirmation by studies on larger patient samples including unipolar and bipolar affective disorder. A notable study on the noradrenergic system was performed by Klimek and co-workers who demonstrated decreased levels of the noradrenalin transporter in patients with major depressive illness (Klimek et al, 1997). Another postmortem analysis showed increased density of the $\alpha_{2 \mathrm{~A}}$-adrenoreceptor in the frontal cortex of suicide victims who had depression (Callado et al, 1998). These two results point to a presynaptic noradrenergic deficit in this disorder consistent with the noradrenergic hypothesis of depression (Schidkraut et al, 1965). Neurohistological studies on the noradrenergic system in bipolar disorder are still required.
Despite indirect evidence that dopaminergic systems are involved in the pathogenesis of mood disorders, only one study of this transmitter system has been performed, reporting increased binding of the D1 receptor in the nucleus accumbens of suicide victims with major depression (Bowden et al, 1997). The nucleus accumbens shows intense connectivity with the prefrontal cortex, mainly with its medial part. Since structural changes in telencephalic basal ganglia as well as in the prefrontal cortex have been shown in patients suffering from major depression, the chemo-architecture of these closely interconnected brain regions would be of interest in mood disorders.

\section{Neuroendocrinological studies}

Some histological investigations have addressed the central endocrine cell groups in the hypothalamus of patients with mood disorders. Expression and messenger mRNA transcripts of corticotrophin-releasing hormone have been shown to be upregulated in neurons of the hypothalamic paraventricular nucleus (Raadsheer et al, 1995). Moreover, data from the same laboratory found an upregulation of vasopressin- and oxytocin-expressing neurons in the same nucleus (Purba et al, 1996). These findings are consistent with clinical data showing a central overactivation of the hypothalamic-pituitary-adrenal axis in depressive disorders (Arborelius et al, 1999). No differences were seen between major depression and bipolar disorder in these post-mortem studies. The investigated samples, however, were too small to allow general conclusions.

\section{VOLUME MEASURES IN MOOD DISORDERS}

In addition to neurohistological studies, structural imaging data provide evidence for the assumption that mood disorders are not exclusively functional illnesses but also show a diversity of morphological changes (Soares \& Mann, 1997; Drevets et al, 1998; Parashos et al, 1998). Despite modern computed tomography and MRI techniques, resolution is still too poor to detect subtle structural alterations of small brain nuclei such as the hypothalamus or some parts of the basal ganglia. A postmortem study was therefore begun to investigate volumes of all telencephalic basal ganglia, of the diencephalon, and of limbic regions, to obtain data on relevant brain circuits in mood disorders using material from our new Magdeburg brain collection. Possible post-mortem artefacts arising from autolysis or tissue processing as well as effects of age and gender were excluded by careful matching of the case. Effects of medication were also controlled for.

The brains of eight patients suffering from major depression or bipolar disorder showed reduced volumes of the left nucleus accumbens, the right putamen and the right and left pallidum externum (Figs $1 \& 2$ ). No differences were seen between bipolar or unipolar depression. The nucleus accumbens was most affected. These data suggest that predominantly limbic-affiliated basal ganglia are involved in the pathology of mood disorders, irrespective of diagnostic polarity (Baumann et al, 1999a).

The brains of patients with major depressive illness showed a trend towards volume reduction for the left amygdala that was not seen in the bipolar disorder patients. Taking into account MRI findings of enlargement of the amygdala in bipolar disorder (Altshuler et al, 1998) and reduced volumes of amygdala core nuclei (Sheline et al, 1998), these data suggest structural differences in the amygdala between major depression and bipolar disorder. The volumes of other limbic regions such as the hippocampus, the temporal horn, the stria terminalis and the basic limbic forebrain were unchanged in our sample.

Volumes of the thalamus and the hypothalamus also showed no differences between the whole patient sample group compared with controls. In major depression, however, the right hypothalamus was smaller than in controls $(P=0.02)$ and the left hypothalamus showed a nonsignificant trend with $13 \%$ smaller volumes $(P=0.07)$.

These post-mortem volume studies confirm and extend previous neuroanatomical models of mood regulation, suggesting a crucial role for ventral parts of telencephalicbasal ganglia and the involvement of the amygdala and hypothalamus in these circuits (for review, see Soares \& Mann, 1997). The preliminary results also show structural differences between major depressive and bipolar disorder for the amygdala.

\section{BR AIN-STEM STUDIES ON MOOD DISORDERS}

Monaminergic (in particular serotonergic and noradrenergic) systems are assumed 
to be essentially involved in the pathology of mood disorders. However, to date there is no information on the possible pathomorphology of the brain-stem nuclei that produce these transmitters. A neurohistological investigation of the dorsal raphe and the locus caeruleus - the main sources of serotonergic and noradrenergic forebrain innervation - was therefore performed.

\section{Locus caeruleus}

Twelve post-mortem brains of patients with mood disorders as well as 12 brains of nonpsychiatric control subjects were included in the study. Six patients had major depressive disorder, the other six bipolar disorder. Based on the topographic organisation of projections from the locus caeruleus (LC), we performed neuron counts separated for the dorsal, medial and ventral portions, as well as the rostral, medial and caudal portions of this nucleus (Baumann et al, 1999b). Surprisingly, total neuron numbers in the whole nucleus were higher in bipolar than unipolar disorder, with this phenomenon being observed on both sides of the LC (Fig. 3). The finding of an enlarged LC in bipolar disorder is consistent with a 'hypernormal' pattern of bipolar disorder morphology comprising larger thalamic volumes (Dupont et al, 1995), greater grey/white ratios (Strakowski et al, 1993), increased gyral complexity (Bullmore $e t a l$, 1994) and enlarged amygdala (Altshuler $e t$ al, 1998; Strakowski et al, 1999).

Topographical analysis revealed that the structural difference is restricted to the rostral two-thirds and the dorsal part of the LC, in which patients with bipolar disorder showed at least a trend to higher neuron numbers compared with patients with major depression or controls. With regard to the topography of LC projections (Mason \& Fibiger, 1979; Loughlin et al, 1982, 1986), it is conceivable that cortical, hippocampal and hypothalamic projections from the LC are part of a morphological substrate for the unipolar-bipolar dichotomy of mood disorders. Together with measurements of noradrenalin or its metabolites in mood disorders (Roy et al, 1985; Azorin et al, 1990), our data indicate that the span between minimum and maximum caerulean noradrenergic transmission in certain brain areas is greater in bipolar disorder than in major depressive disorder. Since noradrenergic tone correlates primarily with clinical symptoms of drive (Carr et al, 1988; Katz et al, 1994; Swann et al,

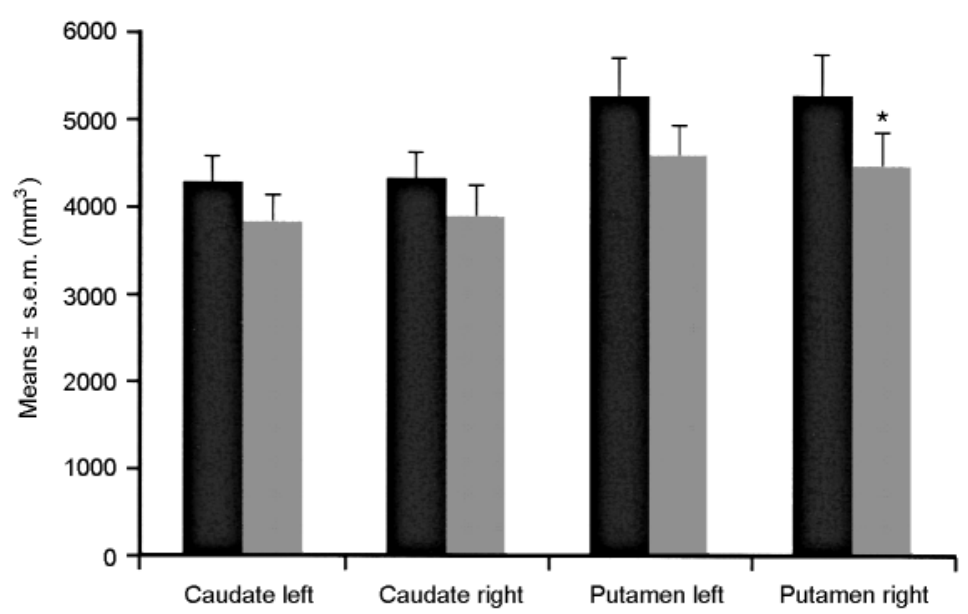

Fig. I Brain structure volumes of patients with affective disorder ( $n=8$, shaded bars) and controls ( $n=8$, black bars) - caudate nucleus and putamen. The volume of the right putamen is reduced in patients. $* P \leqslant 0.05$. Reproduced with permission from Baumann et al (1999a).

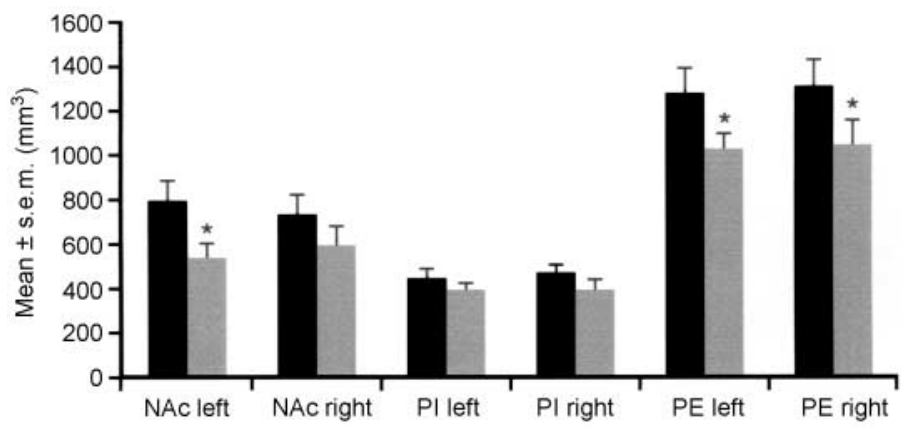

Fig. 2 Brain structure volumes of patients with affective disorder $(n=8$, shaded bars) and controls ( $n=8$, black bars) - nucleus accumbens (NAc), pallidum externum (PE) and pallidum internum (PI). Reduced volumes of the left NAc and both pallida external are seen in patients. $* P \leqslant 0.05$. Reproduced with permission from Baumann et al (1999a).

1994), this wide range of transmission could provide a predisposition for bipolar psychomotor symptoms.

In order to prove noradrenergic function of the LC we performed immunohistochemical analysis of tyrosine hydroxylase, the key enzyme in noradrenalin synthesis (Baumann et al, 1999c). The number of tyrosine hydroxylase-immunoreactive (TH-ir) neurons did not differ between patients with bipolar and unipolar disorder, nor between patients and controls. Further data analysis indicated that suicide might be associated with caerulean noradrenergic function. The number of TH-ir neurons in patients with unipolar or bipolar disorder who died from natural causes was lower than in suicide victims and controls (Fig. 4). This difference remained significant after covarian analysis for mean doses of recent medication with tricyclic or tetracyclic antidepressants. Unipolar and bipolar diagnoses were equally disturbed in the suicide and non-suicide

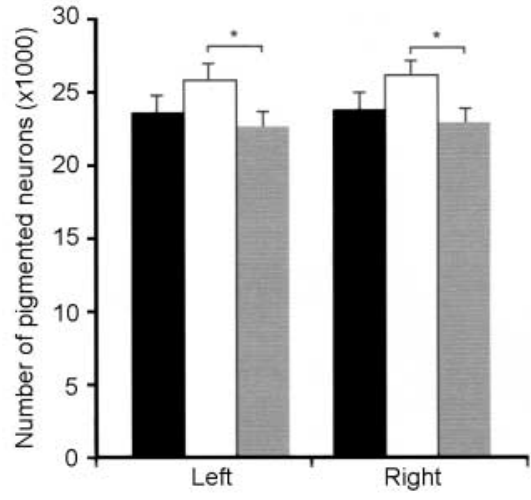

Fig. 3 Pigmented neurons in the locus caeruleus of patients with bipolar disorder ( $n=6$, white bars), major depressive disorder ( $n=6$, shaded bars) and controls (black bars). Reduced neuron numbers are seen in patients. $* P \leqslant 0.05$. Reproduced with permission from Baumann et al (1999b).

group. Numbers of TH-ir neurons correlated positively with mean doses of tricyclic or tetracyclic antidepressants. These results suggest a presynaptic noradrenergic deficit 
of the LC in non-suicidal patients with depression irrespective of unipolar or bipolar diagnosis; they also provide indirect evidence that suicidal behaviour may be related to normalised noradrenergic function, and that traditional antidepressants may enhance noradrenergic activity of the LC in patients with depression.

\section{Dorsal raphe}

The dorsal raphe (DR) exhibits a topographic organisation similar to that of the LC. For example, neuronal projections to the prefrontal cortex and combined projections to the nucleus accumbens and the prefrontal cortex arise from more ventral and rostral subnuclei of the DR (O'Hearn \& Molliver, 1984; Wilson \& Molliver, 1991; Kazakov et al, 1993; Van Bockstaele et al, 1993). Both these projection targets have been implicated in the pathology of depression. Together with the median raphe, the DR provides the ascending serotonergic raphe projections to the forebrain. From these topographic aspects of the raphe system it has been assumed that the DR has a major role in mood regulation (Molliver, 1987). The cyto-architecture of this complex nucleus was investigated in post-mortem brains of 12 patients with mood disorders and 12 subjects without any neuropsychiatric disorder. The ventral parts of the mesencephalic DR (DRvp), i.e. the interfascicular, ventral and ventrolateral subnuclei, showed fewer neurons in patients with a mood disorder (Fig. 5). No differences were seen between major depression and bipolar disorder in this respect. Morphological analysis revealed that the reduction in the number of neurons in patients with a mood disorder was restricted to the ovoid and round neurons.

Since approximately $70 \%$ of the human DR neurons are serotonergic, the lack of neurons in the DRvp is mostly consistent with a regional deficit of serotonergic neurons in mood disorders. Neuronal nucleoli appeared enlarged in the DRvp of patients with mood disorders, suggesting neuronal activation in this area. While antidepressant medication showed no correlation with neuron numbers in any part of the DR, a positive correlation of mean dosage of antidepressant agents with nucleolar size was found for the right ventrolateral subnucleus, which is part of the DRvp.

These results show that patients with a primary mood disorder have a numeric neuronal deficit in the DRvp, irrespective
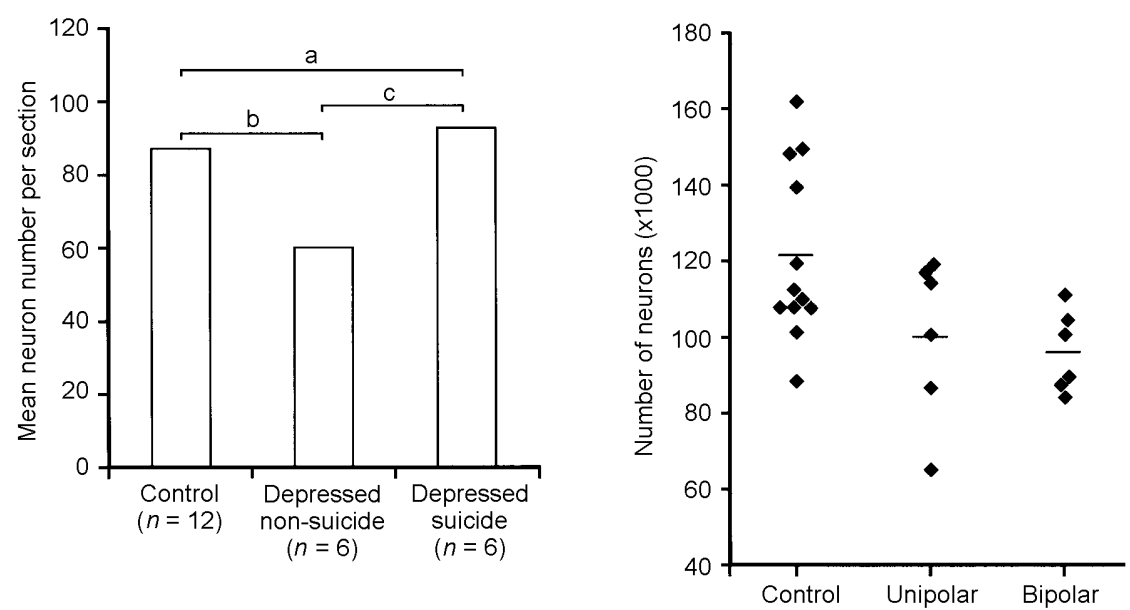

Fig. 4 Tyrosine hydroxylase-immunoreactive neurons in the locus caeruleus. There are fewer immunoreactive neurons in the depressed nonsuicide group compared with the depressed suicide group and controls; a, significant group effect ( $F=4.63$, d.f. $=2,21, P=0.02$ ); b, significant post-hoc test; c, significant post-hoc test. Reproduced with permission from Baumann et al (1999c).

of depressive illness subtype. This structural change may contribute to impaired serotonergic innervation of brain regions that are involved in the pathology of mood disorder. Increased nucleolar size indicating neuronal activation in the DR may reflect an adaptive process partly induced by antidepressant medication.

In order to gain a closer insight into serotonergic function of the DR, tryptophan hydroxylase was investigated by immunodetection in the same sample. Numbers of tryptophan hydroxylaseimmunoreactive (TPH-ir) neurons did not differ between patients and controls, either in the DRvp or in the dorsal or caudal parts of the DR. In the group of patients, however, there was a significant correlation of TPH-ir neurons in the DRvp with mean dosage of antidepressants given in the last 7 days before death $(r=0.69, n=12$, $P=0.01$ ). On the level of single subnuclei, this association was most prominent in the right ventrolateral nucleus. Using antidepressant medication as covariate (ANCOVA), a nearly significant reduction was found for TPH-ir neurons in the DRvp, but not in the dorsal or caudal DR of patients. No difference was observed between major depressive and bipolar disorder samples.

Unchanged serotonin immunohistochemistry and a reduction in Nissl-stained neurons indicate that a neuronal deficit in
Fig. 5 Neuron numbers in ventral subnuclei of the dorsal raphe. Reduced numbers are seen in patients with major depressive disorder (unipolar) and bipolar disorder. The line indicates the mean value for each group.

the DRvp in patients with mood disorders treated by antidepressants is partly compensated for by upregulation of serotonin synthesis as an effect of antidepressant medication. Moreover, our data suggest that in mood disorders, irrespective of diagnostic polarity and apart from medication, synthesis of serotonin may be statedependently reduced in distinct regions of the DR.

\section{CONCLUSION}

There are few post-mortem studies on bipolar disorder, and definite conclusions can hardly be drawn owing to the small and selective nature of the samples, which in particular might produce false-negative results. Moreover, problems sometimes arise from effects of medication or agonal and post-mortem changes. Data obtained from neuroimaging in mood disorders showing structural abnormalities in the frontal and temporal cortices as well as in subcortical regions are widely confirmed, and also extended and specified, by results from neurohistological investigations. Basal ganglia, preferentially those closely associated with the limbic system, have smaller volumes in patients with depressive illness irrespective of diagnostic polarity. Higher neuron numbers in the locus caeruleus of patients with bipolar disorder than in that of patients with major depressive disorder are in agreement with neuroimaging data indicating a 'hypernormal' structural pattern in bipolar disorder that does not 
appear to exist in major depression. Postmortem studies showed no differences between the two types of disorder for noradrenalin and serotonin synthesis in the locus caeruleus and the dorsal raphe. Our data suggest a regionally reduced synthesis of these neurotransmitters, which have a major role in the pathogenesis of mood disorders. Antidepressant medication seems to increase serotonin and noradrenalin production in depressive states and may thereby contribute to normalisation of monaminergic functions.

\section{REFERENCES}

Altshuler, L. L., Casanova, M. F., Goldberg, T. E., et al (1990) The hippocampus and parahippocampus in schizophrenia, suicide, and control brains. Archives of General Psychiatry, 47, 1029-1034.

_ , Bartzokis, G., Grieder, T., et al (1998) Amygdala enlargement in bipolar disorder and hippocampal reduction in schizophrenia: an MRI study demonstrating neuroanatomic specificity. Archives of General Psychiatry, 55, 663-664.

\section{Arango, V., Underwood, M. D. \& Mann, J. J. (1996)} Fewer pigmented locus caeruleus neurons in suicide victims: preliminary results. Biological Psychiatry, 39, 112-120.

Arborelius, L., Owens, M. J., Plotsky, P. M., et a (1999) The role of corticotropin-releasing factor in depression and anxiety disorders. Journal of Endocrinology, 160, I-12.

Azorin, J. M., Pupeschi, G., Valli, M., et al (1990) Plasma 3-methoxy-4-hydroxyphenylglycol in manic patients: relationships with clinical variables. Acto Psychiatrica Scandinavica, 8I, 14-18.

Baumann, B., Danos, P., Krell, D., et al (1999a) Reduced volume of limbic system-affiliated basal ganglia in mood disorders: preliminary data from a postmortem study. Journal of Neuropsychiatry and Clinical Neurosciences, II, 7I-78.

et al (1999b) Unipolar-bipolar dichotomy of mood disorders is supported by noradrenergic brainstem system morphology. Journal of Affective Disorders, 54, 217-224.

_, _ , Diekmann, S., et al (1999c) Impact of suicide and diagnosis on tyrosine hydroxylase expressing neurons in the locus caeruleus of patients with mood disorders. European Archives of Psychiatry and Clinical Neuroscience, 249, 212-219.

Beckmann, H. \& Jakob, H. (1991) Prenatal disturbances of nerve cell migration in the entorhinal region: a common vulnerability factor in functional psychoses? Journal of Neural Transmission General Section 84, $155-164$.

Benes, F. M., Kwok, E.W., Vincent, S. L. et al (1998) A reduction of nonpyramidal cells in sector CA2 of schizophrenics and manic depressives. Biological Psychiatry, 44, 88-97.

Bernstein, H. G., Krell, D., Baumann, B., et al (1998) Morphometric studies of the entorhinal cortex in neuropsychiatric patients and controls: clusters of heterotopically displaced lamina II neurons are not indicative of schizophrenia. Schizophrenia Research, 33 $125-132$

\section{CLINICAL IMPLICATIONS}

Morphological changes in basal ganglia and monaminergic transmitter systems may be vulnerability markers for unipolar disorder.

- Brain biology differs between bipolar and unipolar disorders, suggesting the need for different treatment strategies.

- Effects of antidepressants may be mediated by changes in monaminergic brainstem neurons.

\section{LIMITATIONS}

- Neurohistological research in bipolar disorder is in its infancy and as yet provides only a preliminary assessment of morphological changes involved with this disease.

- Relatively small sample sizes and the high proportion of suicide cases in postmortem studies on mood disorders make it difficult to generalise findings.

It is difficult to correlate morphological findings with acute symptoms.

BRUNO BAUMANN, MD, BERNHARD BOGERTS, MD, Department of Psychiatry, University of Magdeburg, Germany

Correspondence: Dr Bruno Baumann, Department of Psychiatry, University of Magdeburg, Leipziger Strasse 44, Magdeburg D-39120, Germany. Tel: +49 3916715 029; fax: +49 39I 6715 223;

e-mail: baumann@medizin.uni-magdeburg.de

Bowden, C., Theodorou, A. E., Cheetham, S. C., et a (1997) Dopamine DI and D2 receptor binding sites in brain samples from depressed suicides and controls. Brain Research, 752, 227-233.

Brown, R., Colter, N., Corsellis, J. A., et al (1986) Postmortem evidence of structural brain changes in schizophrenia. Differences in brain weight, temporal horn area, and parahippocampal gyrus compared with affective disorder. Archives of General Psychiatry, 43 $36-42$

Bullmore, E., Brammer, M., Harvey, I., et al (1994) Fractal analysis of the boundary between white matter and cerebral cortex in magnetic resonance images: a controlled study of schizophrenic and manic-depressive patients. Psychological Medicine, 24, 77I-78I.

Callado, L. F., Meana, J. J., Grijalba, B., et al (1998) Selective increase of alpha2A-adrenoceptor agonist binding sites in brains of depressed suicide victims. Journal of Neurochemistry, 70, III4-1123.

Carr, V., Edwards, J. \& Prior, M. (1988) Urinary MHPG, platelet ${ }^{3} \mathrm{H}$-imipramine binding and symptomatology in depression: an exploratory study of clinical heterogeneity. Biological Psychiatry, 23, 560-574.

Chan-Palay, V. \& Asan, E. (1989) Alterations in catecholamine neurons of the locus caeruleus in senile dementia of the Alzheimer type and in Parkinson's disease with and without dementia and depression Journal of Comparative Neurology, 287, 373-392.

Diekmann, S., Baumann, B., Schmidt, U., et al (1998) Significant reduction of calretinin-ir neurons in the anterior cingulate cortex in subjects with affective disorder. Society for Neuroscience Abstract, 24, (Part I) 989.
Drevets, W. C., Ongur, D. \& Price, J. L. (1998)

Neuroimaging abnormalities in the subgenual prefrontal cortex: implications for the pathophysiology of familial mood disorders. Molecular Psychiatry, 3, 190-191; 220-226.

Dupont, R. M., Jernigan, T. L., Heindel, W., et al (1995) Magnetic resonance imaging and mood disorders. Localization of white matter and other subcortical abnormalities. Archives of General Psychiatry. 52, 747-755.

Goodwin, G. M. (1997) Neuropsychological and neuroimaging evidence for the involvement of the frontal lobes in depression. Journal of Psychopharmacology, II, 115-122.

Katz, M. M., Maas, J.W., Frazer, A., et al (1994) Drug-induced actions on brain neurotransmitter systems and changes in the behaviors and emotions of depressed patients. Neuropsychopharmacology, II, $89-100$

Kazakov, V. N., Kravtsov, P. Y., Krakhotkina, E. D. et al (1993) Sources of cortical, hypothalamic and spinal serotonergic projections: topical organization within the nucleus raphe dorsalis. Neuroscience, 56, 157-164.

Klimek, V., Stockmeier, C., Overholser, J., et a (1997) Reduced levels of norepinephrine transporters in the locus caeruleus in major depression. Journal of Neuroscience, $17,8451-8458$.

Little, K. Y. McLaughlin, D. P., Ranc, J., et al (1997) Serotonin transporter binding sites and mRNA levels in depressed persons committing suicide. Biological Psychiatry, 4I, II56-1164.

Loughlin, S. E., Foote, S. L. \& Fallon, J. H. (1982) Locus caeruleus projections to cortex: topography, 
morphology and collateralization. Brain Research Bulletin, 9, 287-294.

_, _ \& Bloom, F. E. (1986) Efferent projections of nucleus locus caeruleus: topographic organization of cells of origin demonstrated by three-dimensional reconstruction. Neuroscience, 18, 291-306.

Malison, R. T., Price, L. H., Berman, R., et al (1998) Reduced brain serotonin transporter availability in major depression as measured by [1231]-2 betacarbomethoxy-3 beta-(4-iodophenyl)tropane and single photon emission computed tomography. Biological Psychiatry, 44, 1090-1098.

Mason, S. T. \& Fibiger, H. C. (1979) Regional topography within noradrenergic locus caeruleus as revealed by retrograde transport of horseradish peroxidase. Journal of Comparative Neurology, 187, 703-724.

Molliver, M. E. (1987) Serotonergic neuronal systems: what their anatomic organization tells us about function. Journal of Clinical Psychopharmacology, 7 (suppl. 6), 3-23.

O'Hearn, E. \& Molliver, M. E. (1984) Organization of raphe-cortical projections in rat: a quantitative retrograde study. Brain Research Bulletin, 13, 709-726.

Parashos, I. A., Tupler, L. A., Blitchington, T., et al (1998) Magnetic-resonance morphometry in patients with major depression. Psychiatry Research, 84, 7-15.

Pearlson, G. D., Barta, P. E., Powers, R. E., et al (1997) Medial and superior temporal gyral volumes and cerebral asymmetry in schizophrenia versus bipolar disorder. Biological Psychiatry, 4I, I-14.

Purba, J. S., Hoogendijk, W. J., Hofman, M. A., et al (1996) Increased number of vasopressin- and oxy tocinexpressing neurons in the paraventricular nucleus of the hypothalamus in depression. Archives of General Psychiatry, 53, 137-143.
Raadsheer, F. C., Hoogendijk, W. J., Stam, F. C., et al (1994) Increased numbers of corticotropin-releasing hormone expressing neurons in the hypothalamic paraventricular nucleus of depressed patients. Neuroendocrinology, 60, 436-444.

_, van Heerikhuize, J. J., Lucassen, P. J., et al (1995) Corticotropin-releasing hormone mRNA levels in the paraventricular nucleus of patients with Alzheimer's disease and depression. American journal of Psychiatry I52, 1372-1376.

Raghavan, R., Day, K. A. \& Perry, R. H. (1995) Rapid cycling bipolar affective disorder and familial learning disability associated with temporal lobe (occipitotemporal gyrus) cortical dysplasia. Journal of Intellectual Disability Research, 39, 509-519.

Rajkowska, G. (1997) Morphometric methods for studying the prefrontal cortex in suicide victims and psychiatric patients. Annals of the New York Academy of Science, 836, 253-268.

Roy, A., Pickar, D., Linnoila, M., et al (1985) Plasma norepinephrine level in affective disorders. Relationship to melancholia. Archives of General Psychiatry, 42, |l81-1185.

Roy, P. D., Zipursky, R. B., Saint-Cyr, J. A., et a (1998) Temporal horn enlargement is present in schizophrenia and bipolar disorder. Biological Psychiatry, 44, 418-422.

Schildkraut, J. J. (1965) The catecholamine hypothesis of affective disorders: a review of supporting evidence. American Journal of Psychiatry, 122, 509-522.

Sheline, Y. I., Gado, M. H. \& Price, J. L. (1998) Amygdala core nuclei volumes are decreased in recurrent major depression. Neuroreport, 9, 2023-2028.

Soares, J. C. \& Mann, J. J. (1997) The anatomy of mood disorders - review of structural neuroimaging studies. Biological Psychiatry, 4I, 86-106.
Stockmeier, C. A., Dilley, G. E., Shapiro, L. A., et al (1997) Serotonin receptors in suicide victims with major depression. Neuropsychopharmacology, 16, 162-173.

_ , Shapiro, L. A., Dilley, G. E., et al (1998) Increase in serotonin-IA autoreceptors in the midbrain of suicide victims with major depression - postmortem evidence for decreased serotonin activity. Journal of Neuroscience, 18, 7394-740।.

Strakowski, S. M., Wilson, D. R., Tohen, M., et al (1993) Structural brain abnormalities in first-episode mania. Biological Psychiatry, 33, 602-609.

_, DelBello, M. P., Sax, K. W., et al (1999) Brain magnetic resonance imaging of structural abnormalities in bipolar disorder. Archives of General Psychiatry, 56, 254-260.

Swann, A. C., Stokes, P. E., Secunda, S. K., et al (1994) Depressive mania versus agitated depression: biogenic amine and hypothalamic-pituitaryadrenocortical function. Biological Psychiatry, 35, 803-813.

Van Bockstaele, E. J., Biswas, A. \& Pickel, V. M. (1993) Topography of serotonin neurons in the dorsal raphe nucleus that send axon collaterals to the rat prefrontal cortex and nucleus accumbens. Brain Research, 624, 188-198.

Vincent, S. L., Todtenkopf, M. S. \& Benes, F. M. (1997) A comparison of the density of pyramidal and nonpyramidal neurons in the anterior cingulate cortex of schizophrenics and manic-depressives. Society for Neuroscience Abstract, 23, 2199.

Wilson, M. A. \& Molliver, M. E. (1991) The organization of serotonergic projections to cerebral cortex in primates: retrograde transport studies. Neuroscience, 44, 555-570. 